PROCEEDINGS OF THE

AMERICAN MATHEMATICAL SOCIETY

Volume 132, Number 1, Pages 77-86

S 0002-9939(03)07205-8

Article electronically published on July 28, 2003

\title{
TIGHT WAVELET FRAMES GENERATED BY THREE SYMMETRIC $B$-SPLINE FUNCTIONS WITH HIGH VANISHING MOMENTS
}

\author{
BIN HAN AND QUN MO
}

(Communicated by David R. Larson)

\begin{abstract}
In this note, we show that one can derive from any $B$-spline function of order $m(m \in \mathbb{N})$ an MRA tight wavelet frame in $L_{2}(\mathbb{R})$ that is generated by the dyadic dilates and integer shifts of three compactly supported real-valued symmetric wavelet functions with vanishing moments of the highest possible order $m$.
\end{abstract}

\section{INTRODUCTION}

$B$-spline functions and tight wavelet frames are of great interest in many applications. The $B$-spline function of order $m(m \in \mathbb{N})$, denoted by $B_{m}$ throughout this note, can be obtained via the following recursive formula: $B_{1}=\chi_{[0,1]}$, the characteristic function of the interval $[0,1]$, and

$$
B_{m}(x):=\int_{0}^{1} B_{m-1}(x-t) d t, \quad x \in \mathbb{R}, m=2,3, \ldots
$$

The $B$-spline function $B_{m} \in C^{m-2}(\mathbb{R})$ is a function of piecewise polynomials of degree less than $m$, vanishes outside the interval $[0, m]$ and is symmetric about the point $x=m / 2$ (that is, $B_{m}(m-x)=B_{m}(x)$ for all $\left.x \in \mathbb{R}\right)$. It is well known that the $B$-spline function $B_{m}$ is a refinable function satisfying the refinement equation

$$
\widehat{B}_{m}(2 \xi)=\left(\frac{1+e^{-i \xi}}{2}\right)^{m} \widehat{B}_{m}(\xi), \quad \xi \in \mathbb{R}
$$

where the Fourier transform of $f \in L_{1}(\mathbb{R})$ is defined to be $\widehat{f}(\xi):=\int_{\mathbb{R}} f(t) e^{-i t \xi} d t, \xi \in$ $\mathbb{R}$.

Tight wavelet frames can be constructed from refinable functions. We say that a set $\left\{\psi^{1}, \ldots, \psi^{r}\right\}$ of functions in $L_{2}(\mathbb{R})$ generates a (normalized) tight wavelet frame in $L_{2}(\mathbb{R})$ if

$$
\sum_{\ell=1}^{r} \sum_{j \in \mathbb{Z}} \sum_{k \in \mathbb{Z}}\left|\left\langle f, \psi_{j, k}^{\ell}\right\rangle\right|^{2}=\|f\|^{2} \quad \forall f \in L_{2}(\mathbb{R}),
$$

Received by the editors April 9, 2002.

2000 Mathematics Subject Classification. Primary 42C40, 41A15, 41A25.

Key words and phrases. Symmetric tight wavelet frames, B-spline functions, vanishing moments.

Research was supported in part by NSERC Canada under Grant G121210654 and by Alberta Innovation and Science REE under Grant G227120136. 
where $\langle f, g\rangle:=\int_{\mathbb{R}} f(t) \overline{g(t)} d t,\|f\|^{2}:=\langle f, f\rangle$ and $\psi_{j, k}^{\ell}:=2^{j / 2} \psi^{\ell}\left(2^{j} \cdot-k\right)$. The set $\left\{\psi^{1}, \ldots, \psi^{r}\right\}$ is called a set of generators for the tight frame $\left\{\psi_{j, k}^{\ell}: \ell=1\right.$, $\ldots, r ; j, k \in \mathbb{Z}\}$. Throughout this note, we assume that the generators for any tight wavelet frame discussed in this note are real-valued and compactly supported functions in $L_{2}(\mathbb{R})$.

One of the most important properties of a tight wavelet frame is its vanishing moments. A set $\left\{\psi^{1}, \ldots, \psi^{r}\right\}$ of compactly supported functions in $L_{2}(\mathbb{R})$ is said to have vanishing moments of order $n$ if

$$
\int_{\mathbb{R}} \psi^{\ell}(t) t^{k} d t=0, \quad \ell=1, \ldots, r ; k=0, \ldots, n-1 .
$$

Recently, there is a growing interest in constructing MRA tight wavelet frames derived from refinable functions, in particular, derived from the $B$-spline functions $B_{m}(m \in \mathbb{N})$ (see [1], [2], [4], [5], [8], [9]). For example, Ron and Shen in [9] demonstrated that a tight wavelet frame with $m$ symmetric generators with short support can be derived from the $B$-spline function $B_{m}(m \in \mathbb{N})$. Recently, Chui and $\mathrm{He}$ in [1] further showed that a tight wavelet frame with only 3 symmetric generators (or 2 generators without symmetry) of compact support can be derived from the $B$-spline function $B_{m}(m \in \mathbb{N})$. Though the constructions in [1], [9] are very interesting, such tight wavelet frames derived from the $B$-spline functions in [1], 9] can have vanishing moments of order no more than one ([2, [4]). It is known that in certain applications the order of vanishing moments of a tight wavelet frame is very important. In order to achieve high order of vanishing moments, very recently, Chui, He and Stöckler [2] and Daubechies, Han, Ron and Shen 4] proposed a very interesting method to derive MRA wavelet tight frames from refinable functions. In particular, it was shown in [2, 4] that a tight wavelet frame with 2 generators of compact support can be derived from any $B$-spline function $B_{m}(m \in \mathbb{N})$ such that it can have vanishing moments of the highest possible order $m$. Unfortunately, except for very few cases, the tight wavelet frames with 2 generators derived from $B$-spline functions in [2], 4] are not symmetric. It is desirable to have symmetric tight wavelet frames with high vanishing moments in applications. On the other hand, it was shown in Petukhov [8] that it is not possible to construct tight wavelet frames with 2 symmetric generators of compact support from the $B$-spline functions $B_{m}(1 \leqslant m \leqslant 100, m \neq 1,2,3,7)$ by using the unitary extension principle in [9].

All the above known results naturally lead to the following interesting question:

Question. Can one obtain a tight wavelet frame with 3 symmetric generators of compact support from any given $B$-spline function $B_{m}(m \in \mathbb{N})$ of order $m$ such that the symmetric tight wavelet frame can have the vanishing moments of the highest possible order $m$ ?

By numerical computation, it was possible to verify in 4] (also see 2]) that the above question is true for $m=1,2,4$, and 6 . It is the purpose of this note to completely settle the above question. The following is the main result of this note.

Theorem 1. Let $m$ be a positive integer and let $B_{m}$ denote the B-spline function of order $m$ which is defined in (1.1). Then there exist three finitely supported sequences $b^{1}, b^{2}, b^{3}$ on $\mathbb{Z}$, which can be easily constructed by a simple procedure, such that by defining

$$
\psi^{\ell}:=\sum_{k \in \mathbb{Z}} b^{\ell}(k) B_{m}(2 \cdot-k), \quad \ell=1,2,3,
$$


one has

(1) $\left\{\psi^{1}, \psi^{2}, \psi^{3}\right\}$ generates a tight wavelet frame in $L_{2}(\mathbb{R})$ and has the vanishing moments of order $m$;

(2) $\psi^{1}, \psi^{2}, \psi^{3}$ are real-valued, symmetric and compactly supported functions such that

$\psi^{1}(1-t)=(-1)^{m} \psi^{1}(t), \quad \psi^{2}(m-t)=\psi^{2}(t), \quad \psi^{3}(m-t)=-\psi^{3}(t), \quad t \in \mathbb{R}$.

The following is an outline of the note. In Section 2, we shall establish some auxiliary results. In Section 3, we shall prove Theorem 1 Also in Section 3, we shall give a step-by-step simple procedure for deriving the sequences $b^{1}, b^{2}, b^{3}$ in Theorem 1

\section{Auxiliary Results}

In order to prove Theorem 1, let us introduce some auxiliary results in this section which are of interest in their own right.

The following lemma can be easily verified.

Lemma 2. If $a_{j} \geqslant 0$ and $b_{j}>0$ for all $j=1, \ldots, n$ such that $\frac{a_{1}}{b_{1}} \geqslant \frac{a_{2}}{b_{2}} \geqslant \cdots \geqslant \frac{a_{n}}{b_{n}}$, then

$$
\frac{a_{1}}{b_{1}} \geqslant \frac{a_{1}+a_{2}}{b_{1}+b_{2}} \geqslant \cdots \geqslant \frac{a_{1}+a_{2}+\cdots+a_{n}}{b_{1}+b_{2}+\cdots+b_{n}} .
$$

For any positive integer $m$, throughout this note, we define

$$
c_{0}:=1 \quad \text { and } \quad c_{j}:=\frac{(2 j-1) ! !}{(2 j) ! !(2 j+1)}=\frac{1}{2 j+1} \prod_{k=1}^{j}\left(1-\frac{1}{2 k}\right), \quad j \in \mathbb{N} .
$$

Note that

$$
\frac{\xi / 2}{\sin (\xi / 2)}=\frac{\arcsin (\sin (\xi / 2))}{\sin (\xi / 2)}=\sum_{j=0}^{\infty} c_{j} \sin ^{2 j} \frac{\xi}{2}, \quad \xi \in[-\pi, \pi] .
$$

Clearly, we have

$$
\sum_{j=0}^{\infty} c_{j}=\frac{\pi}{2}
$$

The following estimate will be needed later.

Lemma 3. Let $c_{j}(j \in \mathbb{N} \cup\{0\})$ be defined in (2.1). For any positive integer $m$, define

$$
f_{m}(x):=4 m(1+x)^{2 m} \sum_{j=0}^{\infty} c_{m+j}\left(1-x^{2}\right)^{j}, \quad x \in[0,1] .
$$

Then for $m \geqslant 3, f_{m}$ is an increasing function on the interval $[0,1]$ and

$$
f_{m}(x) \geqslant \frac{\pi}{1-2^{1 / 2-m}} \quad \forall x \in[0,1] .
$$

Proof. By (2.3), it is easy to see that $f_{m}$ is a continuous function on $[0,1]$. For $x \in(0,1)$, by computation, we have

$f_{m}^{\prime}(x)=8 m^{2}(1+x)^{2 m-1} \sum_{j=0}^{\infty} c_{m+j}\left(1-x^{2}\right)^{j}-8 m(1+x)^{2 m} x \sum_{j=0}^{\infty} j c_{m+j}\left(1-x^{2}\right)^{j-1}$. 
Consequently,

$\frac{f_{m}^{\prime}(x)}{8 m(1+x)^{2 m} x}=m \frac{x^{-1}-1}{1-x^{2}} \sum_{j=0}^{\infty} c_{m+j}\left(1-x^{2}\right)^{j}-\sum_{j=0}^{\infty} j c_{m+j}\left(1-x^{2}\right)^{j-1}, \quad x \in(0,1)$.

Denote $y:=1-x^{2}$. Then

$$
\frac{1}{x}=\frac{1}{\sqrt{1-y}}=(1-y)^{-1 / 2}=1+\sum_{j=1}^{\infty}(2 j+1) c_{j} y^{j}, \quad x \in(0,1) .
$$

Therefore, for $x \in(0,1)$, we have

$$
\begin{aligned}
\frac{f_{m}^{\prime}(x)}{8 m(1+x)^{2 m} x} & =m\left(\sum_{j=0}^{\infty}(2 j+3) c_{j+1} y^{j}\right)\left(\sum_{j=0}^{\infty} c_{m+j} y^{j}\right)-\sum_{j=0}^{\infty}(j+1) c_{m+j+1} y^{j} \\
& =\sum_{j=0}^{\infty} g_{m, j} c_{m+j+1} y^{j}
\end{aligned}
$$

where $y=1-x^{2}$ and the numbers $g_{m, j}$ are defined by

$$
g_{m, j}:=m \sum_{k=0}^{j}(2 k+3) c_{k+1} \frac{c_{m+j-k}}{c_{m+j+1}}-(j+1), \quad j \in \mathbb{N} \cup\{0\} .
$$

By the definition of the numbers $c_{j}(j \in \mathbb{N} \cup\{0\})$ in (2.1), we have

$$
(2 k+3) c_{k+1} \frac{c_{m+j-k}}{c_{m+j+1}}=\frac{2 m+2 j+3}{2 m+2 j-2 k+1} \frac{\prod_{\ell=1}^{k+1}\left(1-\frac{1}{2 \ell}\right)}{\prod_{\ell=m+j-k+1}^{m+j+1}\left(1-\frac{1}{2 \ell}\right)} .
$$

Note that for any nonnegative integer $k$, we have

$$
\begin{aligned}
\prod_{\ell=1}^{k+1}\left(1-\frac{1}{2 \ell}\right) & =\frac{1}{2} \prod_{\ell=2}^{k+1}\left(1-\frac{1}{2 \ell}\right) \geqslant \frac{1}{2} \prod_{\ell=2}^{k+1} \sqrt{1-\frac{1}{2 \ell}} \sqrt{1-\frac{1}{2 \ell-1}} \\
& =\frac{1}{2} \prod_{\ell=2}^{k+1} \sqrt{\frac{\ell-1}{\ell}}=\frac{1}{2 \sqrt{k+1}}
\end{aligned}
$$

and for $0 \leqslant k \leqslant j$,

$$
\begin{aligned}
\prod_{\ell=m+j-k+1}^{m+j+1}\left(1-\frac{1}{2 \ell}\right) & \leqslant \prod_{\ell=m+j-k+1}^{m+j+1} \sqrt{1-\frac{1}{2 \ell}} \sqrt{1-\frac{1}{2 \ell+1}} \\
& =\prod_{\ell=m+j-k+1}^{m+j+1} \frac{\sqrt{2 \ell-1}}{\sqrt{2 \ell+1}}=\frac{\sqrt{2 m+2 j-2 k+1}}{\sqrt{2 m+2 j+3}} .
\end{aligned}
$$

It follows from (2.6) and the above two inequalities that

$$
\begin{aligned}
(2 k+3) c_{k+1} \frac{c_{m+j-k}}{c_{m+j+1}} & \geqslant \frac{2 m+2 j+3}{2 m+2 j-2 k+1} \cdot \frac{1}{2 \sqrt{k+1}} \cdot \frac{\sqrt{2 m+2 j+3}}{\sqrt{2 m+2 j-2 k+1}} \\
& =\frac{1}{2 \sqrt{k+1}} \frac{(m+j+3 / 2)^{3 / 2}}{(m+j+1 / 2-k)^{3 / 2}} .
\end{aligned}
$$


Hence,

$$
\begin{aligned}
g_{m, j}+(j+1) & =m \sum_{k=0}^{j}(2 k+3) c_{k+1} \frac{c_{m+j-k}}{c_{m+j+1}} \\
& \geqslant m(m+j+3 / 2)^{3 / 2} \sum_{k=0}^{j} \frac{1}{2 \sqrt{k+1}(m+j+1 / 2-k)^{3 / 2}} \\
& =m(m+j+3 / 2)^{3 / 2} \sum_{k=1}^{j+1} \frac{1}{2 \sqrt{k}(m+j+3 / 2-k)^{3 / 2}} .
\end{aligned}
$$

Let $t_{m, j, k}^{1}:=\sqrt{(k+1)(m+j+5 / 2-k)}$ and $t_{m, j, k}^{2}:=\sqrt{k(m+j+5 / 2-(k+1))}$. Then $t_{m, j, k}^{1} \geqslant \sqrt{k} \sqrt{m+j+3 / 2-k}=t_{m, j, k}^{2}$. We have

$$
\begin{aligned}
\frac{\sqrt{k+1}}{\sqrt{m+j+5 / 2-(k+1)}} & -\frac{\sqrt{k}}{\sqrt{m+j+5 / 2-k}} \\
& =\frac{t_{m, j, k}^{1}-t_{m, j, k}^{2}}{\sqrt{m+j+5 / 2-(k+1)} \sqrt{m+j+5 / 2-k}} \\
& =\frac{(k+1)(m+j+5 / 2-k)-k(m+j+5 / 2-(k+1))}{\sqrt{m+j+3 / 2-k} \sqrt{m+j+5 / 2-k}\left(t_{m, j, k}^{1}+t_{m, j, k}^{2}\right)} \\
& =\frac{m+j+5 / 2}{\sqrt{m+j+3 / 2-k} \sqrt{m+j+5 / 2-k}\left(t_{m, j, k}^{1}+t_{m, j, k}^{2}\right)} \\
& \leqslant \frac{m+j+5 / 2}{2 \sqrt{k}(m+j+3 / 2-k)^{3 / 2}} .
\end{aligned}
$$

We deduce that

$$
\begin{aligned}
g_{m, j}+(j+1) & \frac{m(m+j+3 / 2)^{3 / 2}}{m+j+5 / 2} \sum_{k=1}^{j+1}\left[\frac{\sqrt{k+1}}{\sqrt{m+j+5 / 2-(k+1)}}\right. \\
= & \left.-\frac{\sqrt{k}}{\sqrt{m+j+5 / 2-k}}\right] \\
= & \frac{m(m+j+3 / 2)^{3 / 2}}{m+j+5 / 2}\left[\frac{\sqrt{j+2}}{\left.\sqrt{m+1 / 2}-\frac{\sqrt{1}}{\sqrt{m+j+3 / 2}}\right]}\right] \\
& \left.\times \frac{m+j+3 / 2)^{3 / 2}}{\sqrt{m+j+5 / 2}}\right] \\
= & \frac{m(j+2)(m+j+3 / 2)-(m+1 / 2)}{\sqrt{(m+1 / 2)(j+2)(m+j+3 / 2)} \sqrt{m+j+3 / 2}(\sqrt{(j+2)(m+j+3 / 2)}+\sqrt{m+1 / 2})}
\end{aligned}
$$


By computation, for $m \geqslant 2$ and $j \geqslant 0$, we have

$$
\begin{aligned}
{[m(m+j+3 / 2)-} & m-1 / 2]^{2}-(m+1 / 2)(j+2)(m+j+3 / 2) \\
=[ & m(m-1)-1 / 2] j^{2}+\left[m\left(2 m^{2}-5\right)-7 / 4\right] j \\
& \quad+m(m+1)\left(m^{2}-4\right)+\frac{5}{4} m(m-1)+\frac{3}{4}\left(m-\frac{5}{3}\right)>0 .
\end{aligned}
$$

Consequently, we have

$m(m+j+3 / 2)>\sqrt{(m+1 / 2)(j+2)(m+j+3 / 2)}+(m+1 / 2) \quad \forall m \geqslant 2, j \geqslant 0$.

Hence, $g_{m, j}+(j+1)>j+1$ for all $m \geqslant 2$ and $j \geqslant 0$. That is,

$$
g_{m, j}>0 \quad \forall m \geqslant 2 \text { and } j \geqslant 0,
$$

which implies that $f_{m}^{\prime}(x)>0$ for all $x \in(0,1)$ and $m \geqslant 2$.

So, for $m \geqslant 2, f_{m}$ is an increasing function on the interval $[0,1]$ and

$$
f_{m}(x) \geqslant f_{m}(0) \quad \forall x \in[0,1] .
$$

Note that $1-\frac{1}{2 k}=\frac{2 k-1}{2 k} \geqslant \sqrt{\frac{k-1}{k}}$ for all $k \in \mathbb{N}$. We deduce that

$$
\begin{aligned}
c_{j} & =\frac{1}{2 j+1} \prod_{k=1}^{j}\left(1-\frac{1}{2 k}\right) \geqslant \frac{1}{2(2 j+1)} \prod_{k=2}^{j} \sqrt{\frac{k-1}{k}} \\
& =\frac{1}{(2 j+1) \sqrt{4 j}} \geqslant \frac{1}{2 \sqrt{j}}-\frac{1}{2 \sqrt{j+1}}
\end{aligned}
$$

for all $j \in \mathbb{N}$. When $m \geqslant 4$, by (2.7), we have

$$
f_{m}(0)=4 m \sum_{j=m}^{\infty} c_{j} \geqslant 4 m \sum_{j=m}^{\infty}\left(\frac{1}{2 \sqrt{j}}-\frac{1}{2 \sqrt{j+1}}\right)=2 \sqrt{m} \geqslant \frac{\pi}{1-2^{1 / 2-m}}
$$

since $h(t):=2 \sqrt{t}\left(1-2^{1 / 2-t}\right), t \geqslant 1$ is an increasing function and $h(4)=4-2^{-3 / 2}>$ $\pi$.

Note that $c_{0}=1, c_{1}=1 / 6$ and $c_{2}=3 / 40$. When $m=3$, by (2.3), we have

$$
f_{3}(0)=12 \sum_{j=0}^{\infty} c_{3+j}=12\left(\frac{\pi}{2}-c_{0}-c_{1}-c_{2}\right)=6 \pi-\frac{149}{10} \geqslant \frac{\pi}{1-2^{1 / 2-3}},
$$

which completes the proof.

Now the main result in this section, which plays a critical role in our proof of Theorem 1 is as follows:

Theorem 4. For any positive integer $m$,

$$
\frac{\sum_{j=0}^{m-1} c_{j} \sin ^{2 j} \frac{\xi}{2}}{\sum_{j=0}^{m-1} c_{j} \sin ^{2 j} \xi} \geqslant\left(\cos ^{2 m} \frac{\xi}{2}+\sin ^{2 m} \frac{\xi}{2}\right)^{\frac{1}{2 m}} \quad \forall \xi \in \mathbb{R},
$$

where the numbers $c_{j}(j \in \mathbb{N} \cup\{0\})$ are defined in (2.1).

Proof. It is easy to see that in order to show the inequality (2.8), it suffices to prove it for $\xi \in[0, \pi / 2]$ since $\sin ^{2} \frac{\pi-\xi}{2}=\cos ^{2} \frac{\xi}{2} \geqslant \sin ^{2} \frac{\xi}{2}$ for all $\xi \in[0, \pi / 2]$. Let $x=\cos (\xi)$. By Lemma 3, for $m \geqslant 3$, we have the following estimate:

$$
4 m\left(2 \cos ^{2} \frac{\xi}{2}\right)^{2 m} \sum_{j=0}^{\infty} c_{m+j} \sin ^{2 j} \xi \geqslant \frac{\pi}{1-2^{1 / 2-m}} \quad \forall \xi \in[0, \pi / 2] .
$$


Define

$$
A(\xi):=\sum_{j=0}^{m-1} c_{j} \sin ^{2 j} \frac{\xi}{2} \quad \text { and } \quad B(\xi):=\frac{\xi / 2}{\sin (\xi / 2)}-A(\xi) .
$$

By (2.2),$B(\xi)=\sum_{j=m}^{\infty} c_{j} \sin ^{2 j} \frac{\xi}{2}$. It follows from (2.9) that for $\xi \in[0, \pi / 2]$,

$$
\begin{aligned}
B(2 \xi) & =\sum_{j=m}^{\infty} c_{j} \sin ^{2 j} \xi=(\sin \xi)^{2 m} \sum_{j=0}^{\infty} c_{m+j} \sin ^{2 j} \xi \\
& =\frac{1}{4 m} \cdot \frac{\sin ^{2 m}(\xi / 2)}{\cos ^{2 m}(\xi / 2)} \cdot 4 m\left(2 \cos ^{2} \frac{\xi}{2}\right)^{2 m} \sum_{j=0}^{\infty} c_{m+j} \sin ^{2 j} \xi \\
& \geqslant \frac{\frac{\pi}{2} \cdot \frac{1}{2 m} \cdot \frac{\sin ^{2 m}(\xi / 2)}{\cos ^{2 m}(\xi / 2)}}{1-2^{1 / 2-m}} \\
& \geqslant \frac{\xi}{\sin \xi} \cdot \frac{\frac{1}{2 m} \cdot \frac{\sin ^{2 m}(\xi / 2)}{\cos ^{2 m}(\xi / 2)}}{1-\frac{1}{\left[4 \cos ^{2}(\xi / 2)\right]^{m}} \cdot \frac{1}{\cos (\xi / 2)}},
\end{aligned}
$$

where we have used the fact that $\cos (\xi / 2) \geqslant 2^{-1 / 2}$ for all $\xi \in[0, \pi / 2]$. Observe that $(1+x)^{\frac{1}{2 m}}-1 \leqslant \frac{x}{2 m}$ for all $x \geqslant 0$ and $m \in \mathbb{N}$. It follows that

$$
\left(1+\frac{\sin ^{2 m}(\xi / 2)}{\cos ^{2 m}(\xi / 2)}\right)^{\frac{1}{2 m}}-1 \leqslant \frac{1}{2 m} \cdot \frac{\sin ^{2 m}(\xi / 2)}{\cos ^{2 m}(\xi / 2)}, \quad \xi \in[0, \pi / 2] .
$$

Since $A(2 \xi)+B(2 \xi)=\frac{\xi}{\sin \xi}, \xi \in[0, \pi / 2]$, from the above two inequalities we have

$$
\begin{aligned}
\frac{B(2 \xi)}{A(2 \xi)+B(2 \xi)}=\frac{B(2 \xi)}{\frac{\xi}{\sin \xi}} \geqslant \frac{\left(1+\frac{\sin ^{2 m}(\xi / 2)}{\cos ^{2 m}(\xi / 2)}\right)^{\frac{1}{2 m}}-1}{\left(1+\frac{\sin ^{2 m}(\xi / 2)}{\cos ^{2 m}(\xi / 2)}\right)^{\frac{1}{2 m}}-\frac{1}{\left[4 \cos ^{2}(\xi / 2)\right]^{m}} \cdot \frac{1}{\cos (\xi / 2)}} \\
=\frac{\left(\cos ^{2 m}(\xi / 2)+\sin ^{2 m}(\xi / 2)\right)^{\frac{1}{2 m}}-\cos (\xi / 2)}{\left(\cos ^{2 m}(\xi / 2)+\sin ^{2 m}(\xi / 2)\right)^{\frac{1}{2 m}}-\frac{1}{\left[4 \cos ^{2}(\xi / 2)\right]^{m}}} .
\end{aligned}
$$

The above inequality is equivalent to

$$
\frac{\cos \frac{\xi}{2}-\frac{B(2 \xi)}{A(2 \xi)+B(2 \xi)} \cdot \frac{1}{\left[4 \cos ^{2}(\xi / 2)\right]^{m}}}{1-\frac{B(2 \xi)}{A(2 \xi)+B(2 \xi)}} \geqslant\left(\cos ^{2 m}(\xi / 2)+\sin ^{2 m}(\xi / 2)\right)^{\frac{1}{2 m}} .
$$

Since $2 \cos (\xi / 2)>1$ for all $\xi \in[0, \pi / 2]$ and

$$
\frac{c_{j} \sin ^{2 j} \frac{\xi}{2}}{c_{j} \sin ^{2 j} \xi}=\frac{1}{\left(2 \cos \frac{\xi}{2}\right)^{2 j}} \geqslant \frac{1}{\left(2 \cos \frac{\xi}{2}\right)^{2 j+2}}=\frac{c_{j+1} \sin ^{2 j+2} \frac{\xi}{2}}{c_{j+1} \sin ^{2 j+2} \xi}, \quad \xi \in[0, \pi / 2],
$$

by Lemma 2 we have

$$
\frac{B(\xi)}{B(2 \xi)}=\frac{\sum_{j=m}^{\infty} c_{j} \sin ^{2 j} \frac{\xi}{2}}{\sum_{j=m}^{\infty} c_{j} \sin ^{2 j} \xi} \leqslant \frac{\sin ^{2 m} \frac{\xi}{2}}{\sin ^{2 m} \xi}=\frac{1}{\left[4 \cos ^{2}(\xi / 2)\right]^{m}}, \quad \xi \in[0, \pi / 2] .
$$


Hence, $B(\xi) \leqslant \frac{B(2 \xi)}{\left[4 \cos ^{2}(\xi / 2)\right]^{m}}$ for all $\xi \in[0, \pi / 2]$ and

$$
\begin{aligned}
\frac{A(\xi)}{A(2 \xi)} & =\frac{[A(\xi)+B(\xi)]-B(\xi)}{[A(2 \xi)+B(2 \xi)]-B(2 \xi)} \geqslant \frac{[A(\xi)+B(\xi)]-\frac{B(2 \xi)}{\left[4 \cos ^{2}(\xi / 2)\right]^{m}}}{[A(2 \xi)+B(2 \xi)]-B(2 \xi)} \\
& =\frac{\frac{A(\xi)+B(\xi)}{A(2 \xi)+B(2 \xi)}-\frac{B(2 \xi)}{A(2 \xi)+B(2 \xi)} \cdot \frac{1}{\left[4 \cos ^{2}(\xi / 2)\right]^{m}}}{1-\frac{B(2 \xi)}{A(2 \xi)+B(2 \xi)}} \\
& =\frac{\cos \frac{\xi}{2}-\frac{B(2 \xi)}{A(2 \xi)+B(2 \xi)} \cdot \frac{1}{\left[4 \cos ^{2}(\xi / 2)\right]^{m}}}{1-\frac{B(2 \xi)}{A(2 \xi)+B(2 \xi)}}
\end{aligned}
$$

since $A(\xi)+B(\xi)=\frac{\xi / 2}{\sin (\xi / 2)}$ for $\xi \in[-\pi, \pi]$. It follows from the above inequality and (2.10) that for $\xi \in[0, \pi / 2]$,

$$
\frac{A(\xi)}{A(2 \xi)} \geqslant \frac{\cos \frac{\xi}{2}-\frac{B(2 \xi)}{A(2 \xi)+B(2 \xi)} \cdot \frac{1}{\left[4 \cos ^{2}(\xi / 2)\right]^{m}}}{1-\frac{B(2 \xi)}{A(2 \xi)+B(2 \xi)}} \geqslant\left(\cos ^{2 m}(\xi / 2)+\sin ^{2 m}(\xi / 2)\right)^{\frac{1}{2 m}} .
$$

Therefore, (2.8) holds for $m \geqslant 3$. It is obvious that (2.8) holds for $m=1$. In the following, let us check the case $m=2$. Let $x=\sin ^{2} \frac{\xi}{2}$. Then $\sin ^{2} \xi=4 x(1-x)$. When $m=2$, to prove (2.8) it suffices to prove

$$
\frac{1+\frac{x}{6}}{1+\frac{2}{3} x(1-x)} \geqslant[1-2 x(1-x)]^{1 / 4} \quad \forall x \in[0,1] .
$$

By computation, for $x \in[0,1]$, we have

$$
\begin{aligned}
{\left[1+\frac{2}{3} x(1-x)\right][1-2 x(1-x)]^{1 / 4} } & \leqslant\left[1+\frac{2}{3} x(1-x)\right]\left[1-\frac{1}{4} 2 x(1-x)\right] \\
& =1+\left(\frac{2}{3}-\frac{1}{2}\right) x(1-x)-\frac{1}{3} x^{2}(1-x)^{2} \\
& \leqslant 1+\frac{1}{6} x(1-x) \\
& \leqslant 1+\frac{1}{6} x,
\end{aligned}
$$

which verifies (2.11). Therefore, the proof is completed.

\section{Proof of the main Result}

In this section, using the auxiliary results in Section 2, we shall prove Theorem 1 In particular, we shall give a step-by-step procedure for constructing the sequences $b^{1}, b^{2}, b^{3}$ in Theorem [1]

The symbol of a sequence $a$ on $\mathbb{Z}$ is defined to be

$$
\widehat{a}(\xi):=\sum_{k \in \mathbb{Z}} a(k) e^{-i k \xi}, \quad \xi \in \mathbb{R} .
$$

Proof of Theorem 1: Let $B_{m}$ be the $B$-spline function of order $m$ and let $\widehat{a}(\xi):=$ $\left(\frac{1+e^{-i \xi}}{2}\right)^{m}$. Then it is known that $\widehat{B}_{m}(2 \xi)=\widehat{a}(\xi) \widehat{B}_{m}(\xi)$. Let $c_{j}(j \in \mathbb{N} \cup\{0\})$ be the numbers that are defined in (2.1). We define the numbers $d_{m, j}(m \in \mathbb{N}, j \in \mathbb{N} \cup\{0\})$, 
which are uniquely determined by the following identity:

$$
\left(\sum_{j=0}^{m-1} c_{j} x^{j}\right)^{m}=\sum_{j=0}^{\infty} d_{m, j} x^{j}, \quad x \in \mathbb{R} .
$$

Clearly, $d_{m, 0}=\left[c_{0}\right]^{m}=1$ and $d_{m, j}=0$ for all $j>m(m-1)$. Define two $2 \pi$-periodic trigonometric polynomials $\theta_{1}$ and $\theta$ as follows:

$$
\theta_{1}(\xi):=1+\sum_{j=1}^{m-1} d_{m, j} \sin ^{2 j} \frac{\xi}{2} \quad \text { and } \quad \theta(\xi):=\left|\theta_{1}(\xi)\right|^{2}=\left[\theta_{1}(\xi)\right]^{2} .
$$

Since $\frac{\sin ^{2}(\xi / 2)}{\sin ^{2} \xi}=\frac{1}{4 \cos ^{2}(\xi / 2)} \leqslant \frac{1}{2}$ for $\xi \in[0, \pi / 2]$, we have $\left(\frac{\sin ^{2}(\xi / 2)}{\sin ^{2} \xi}\right)^{j-1} \geqslant\left(\frac{\sin ^{2}(\xi / 2)}{\sin ^{2} \xi}\right)^{j}$ for all $j \in \mathbb{N}$ and for all $\xi \in[0, \pi / 2]$. By Lemma 2 for $\xi \in[0, \pi / 2]$, we have

$$
\begin{aligned}
\frac{\theta_{1}(\xi)}{\theta_{1}(2 \xi)} & =\frac{\sum_{j=0}^{m-1} d_{m, j} \sin ^{2 j}(\xi / 2)}{\sum_{j=0}^{m-1} d_{m, j} \sin ^{2 j} \xi} \geqslant \frac{\sum_{j=0}^{m(m-1)} d_{m, j} \sin ^{2 j}(\xi / 2)}{\sum_{j=0}^{m(m-1)} d_{m, j} \sin ^{2 j} \xi} \\
& =\left(\frac{\sum_{j=0}^{m-1} c_{j} \sin ^{2 j}(\xi / 2)}{\sum_{j=0}^{m-1} c_{j} \sin ^{2 j} \xi}\right)^{m} .
\end{aligned}
$$

By Theorem 4 in Section 2, for $\xi \in[0, \pi / 2]$ we have

$$
\begin{aligned}
\frac{\theta_{1}(\xi)}{\theta_{1}(2 \xi)} & \geqslant\left(\frac{\sum_{j=0}^{m-1} c_{j} \sin ^{2 j}(\xi / 2)}{\sum_{j=0}^{m-1} c_{j} \sin ^{2 j} \xi}\right)^{m} \geqslant\left(\cos ^{2 m}(\xi / 2)+\sin ^{2 m}(\xi / 2)\right)^{1 / 2} \\
& =\sqrt{|\widehat{a}(\xi)|^{2}+|\widehat{a}(\xi+\pi)|^{2}} .
\end{aligned}
$$

In other words, $\theta(\xi)-\theta(2 \xi)\left(|\widehat{a}(\xi)|^{2}+|\widehat{a}(\xi+\pi)|^{2}\right) \geqslant 0$ for all $\xi \in[0, \pi / 2]$. By the definition of $\theta$, we have $\theta(-\xi)=\theta(\xi)$ and $\theta(\xi) \leqslant \theta(\pi-\xi)$ for all $\xi \in[0, \pi / 2]$ since $d_{m, j} \geqslant 0$ and $\sin ^{2} \frac{\pi-\xi}{2}=\cos ^{2} \frac{\xi}{2} \geqslant \frac{1}{2} \geqslant \sin ^{2} \frac{\xi}{2}$ for all $\xi \in[0, \pi / 2]$. Consequently, we have

$$
\theta(\xi)-\theta(2 \xi)\left(|\widehat{a}(\xi)|^{2}+|\widehat{a}(\xi+\pi)|^{2}\right) \geqslant 0 \quad \forall \xi \in[-\pi, \pi] .
$$

By the Fejér-Riesz lemma, there exists a $2 \pi$-periodic trigonometric polynomial $\theta_{2}$ such that

$$
\left|\theta_{2}(\xi)\right|^{2}=\theta(\xi)-\theta(2 \xi)\left(|\widehat{a}(\xi)|^{2}+|\widehat{a}(\xi+\pi)|^{2}\right) .
$$

Now define

$$
\begin{aligned}
& \widehat{b}^{1}(\xi):=\overline{\widehat{a}(\xi+\pi)} e^{-i \xi} \theta_{1}(2 \xi)=\left(\frac{1-e^{i \xi}}{2}\right)^{m} e^{-i \xi} \theta_{1}(2 \xi), \\
& \widehat{b}^{2}(\xi):=\widehat{a}(\xi)\left[\theta_{2}(2 \xi)+\overline{\theta_{2}(2 \xi)}\right] / 2=\left(\frac{1+e^{-i \xi}}{2}\right)^{m}\left[\theta_{2}(2 \xi)+\overline{\theta_{2}(2 \xi)}\right] / 2, \\
& \widehat{b}^{3}(\xi):=\widehat{a}(\xi)\left[\theta_{2}(2 \xi)-\overline{\theta_{2}(2 \xi)}\right] / 2=\left(\frac{1+e^{-i \xi}}{2}\right)^{m}\left[\theta_{2}(2 \xi)-\overline{\theta_{2}(2 \xi)}\right] / 2 .
\end{aligned}
$$

It is evident that $b^{1}, b^{2}$, and $b^{3}$ are real-valued finitely supported sequences on $\mathbb{Z}$ such that

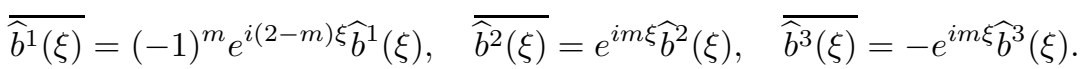

Let

$$
\Theta(\xi):=\theta(2 \xi)\left(|\widehat{a}(\xi)|^{2}+|\widehat{a}(\xi+\pi)|^{2}\right) .
$$


Note that $\left|\theta_{2}(\xi)+\overline{\theta_{2}(\xi)}\right|^{2}+\left|\theta_{2}(\xi)-\overline{\theta_{2}(\xi)}\right|^{2}=4\left|\theta_{2}(\xi)\right|^{2}$. By calculation, we have

$$
\begin{aligned}
|\widehat{a}(\xi)|^{2} \Theta(2 \xi)+ & \left|\widehat{b}^{1}(\xi)\right|^{2}+\left|\widehat{b}^{2}(\xi)\right|^{2}+\left|\widehat{b}^{3}(\xi)\right|^{2} \\
= & |\widehat{a}(\xi)|^{2} \Theta(2 \xi)+|\widehat{a}(\xi+\pi)|^{2}\left|\theta_{1}(2 \xi)\right|^{2}+|\widehat{a}(\xi)|^{2}\left|\theta_{2}(2 \xi)\right|^{2} \\
= & |\widehat{a}(\xi)|^{2} \theta(4 \xi)\left(|\widehat{a}(2 \xi)|^{2}+|\widehat{a}(2 \xi+\pi)|^{2}\right)+|\widehat{a}(\xi+\pi)|^{2} \theta(2 \xi) \\
& \quad+|\widehat{a}(\xi)|^{2}\left[\theta(2 \xi)-\theta(4 \xi)\left(|\widehat{a}(2 \xi)|^{2}+|\widehat{a}(2 \xi+\pi)|^{2}\right)\right] \\
& =\theta(2 \xi)\left(|\widehat{a}(\xi)|^{2}+|\widehat{a}(\xi+\pi)|^{2}\right) \\
& =\Theta(\xi)
\end{aligned}
$$

and

$$
\begin{aligned}
\widehat{a}(\xi) & \overline{\widehat{a}(\xi+\pi)} \Theta(2 \xi)+\widehat{b}^{1}(\xi) \overline{\widehat{b}^{1}(\xi+\pi)}+\widehat{b}^{2}(\xi) \overline{\widehat{b}^{2}(\xi+\pi)}+\widehat{b}^{3}(\xi) \overline{\widehat{b}^{3}(\xi+\pi)} \\
& =\widehat{a}(\xi) \overline{\widehat{a}(\xi+\pi)} \Theta(2 \xi)-\widehat{a}(\xi) \overline{a(\xi+\pi)}\left|\theta_{1}(2 \xi)\right|^{2}+\widehat{a}(\xi) \overline{\widehat{a}(\xi+\pi)}\left|\theta_{2}(2 \xi)\right|^{2} \\
& =\widehat{a}(\xi) \overline{\widehat{a}(\xi+\pi)}\left[\Theta(2 \xi)-\theta(2 \xi)+\left[\theta(2 \xi)-\theta(4 \xi)\left(|\widehat{a}(2 \xi)|^{2}+|\widehat{a}(2 \xi+\pi)|^{2}\right)\right]\right] \\
& =0 .
\end{aligned}
$$

Define $\widehat{\psi}^{\ell}(2 \cdot)=\widehat{b}^{\ell} \widehat{B}_{m}, \ell=1,2,3$. Since $\Theta(0)=1$, by [4, Proposition 1.11] or [2], $\left\{\psi^{1}, \psi^{2}, \psi^{3}\right\}$ generates a tight wavelet frame in $L_{2}(\mathbb{R})$.

It follows from (2.2) that $\theta(\xi)-\theta(2 \xi)|\widehat{a}(\xi)|^{2}=O\left(|\xi|^{2 m}\right), \xi \rightarrow 0$ (also see [4]). Thus, we deduce that $\Theta(\xi)-\Theta(2 \xi)|\widehat{a}(\xi)|^{2}=O\left(|\xi|^{2 m}\right), \xi \rightarrow 0$. Consequently, each wavelet function $\psi^{\ell}, \ell=1,2,3$ has the vanishing moments of order $m$. The symmetry of the wavelet functions $\psi^{1}, \psi^{2}, \psi^{3}$ follows directly from (3.7).

\section{REFERENCES}

[1] C. K. Chui and W. He, Compactly supported tight frames associated with refinable functions, Appl. Comp. Harmonic Anal., 8 (2000), 293-319. MR 2001h:42049

[2] C. K. Chui, W. He, and J. Stöckler, Compactly supported tight and sibling frames with maximum vanishing moments, Appl. Comput. Harmonic Anal., 13 (2002), 224-262.

[3] I. Daubechies, Ten lectures on wavelets, CBMS-NSF Regional Conference Series in Applied Mathematics, 61, SIAM, Philadelphia, 1992. MR 93e:42045

[4] I. Daubechies, B. Han, A. Ron, and Z. W. Shen, Framelets: MRA-based constructions of wavelet frames, Appl. Comput. Harmonic Anal., 14 (2003), No. 1, 1-46.

[5] B. Han, On dual wavelet tight frames, Appl. Comput. Harmonic Anal., 4 (1997), 380-413. MR 98h:42031

[6] D. G. Han and D. R. Larson, Frames, bases and group representations, Mem. Amer. Math. Soc. 147 (2000). MR 2001a:47013

[7] E. Hernández and G. Weiss, A first course on wavelets, CRC Press, Boca Raton, FL, 1996. MR 97i: 42015

[8] A. Petukhov, Symmetric framelets, preprint, (2001).

[9] A. Ron and Z. W. Shen, Affine systems in $L_{2}\left(\mathbb{R}^{d}\right)$ : the analysis of the analysis operator, J. Funct. Anal., 148 (2) (1997), 408-447. MR 99g:42043

Department of Mathematical and Statistical Sciences, University of Alberta, Edmonton, Alberta, Canada T6G 2G1

E-mail address: bhan@math.ualberta.ca

$U R L:$ http://www. ualberta.ca/ bhan

Department of Mathematical and Statistical Sciences, University of Alberta, Edmonton, Alberta, Canada T6G 2G1

E-mail address: mo@math.ualberta.ca

$U R L:$ http://www. math.ualberta.ca/ mo 